\title{
Bioinformatic Analysis of Differential Genes in Sinusoidal Endothelial Cells of Liver Cirrhosis Rat
}

\author{
Xiong Zhao, Haofeng Lu, Mi Li* \\ Department of Hepatobiliary Surgery, The First Affiliated Hospital of Yangtze University, Jingzhou, China \\ Email: ^850489632@qq.com, *limi9453@163.com
}

How to cite this paper: Zhao, X., Lu, H.F. and Li, M. (2021) Bioinformatic Analysis of Differential Genes in Sinusoidal Endothelial Cells of Liver Cirrhosis Rat. Yangtze Medicine, 5, 133-140. https://doi.org/10.4236/ym.2021.52014

Received: October 1, 2020

Accepted: April 12, 2021

Published: April 15, 2021

Copyright ( 2021 by author(s) and Scientific Research Publishing Inc. This work is licensed under the Creative Commons Attribution International License (CC BY 4.0).

http://creativecommons.org/licenses/by/4.0/

\section{(c) (i) Open Access}

\begin{abstract}
Objective: Based on the GEO database, the differential genes of sinusoidal endothelial cells in cirrhotic rats were analyzed. Methods: In the GEO database, the differential gene expressions of liver sinusoidal endothelial cells in cirrhotic rats were obtained. The screening was performed according to $\mathrm{P}<$ 0.01 and differential multiple factor $\geq 4$. The obtained genes were input into the DAVID database for enrichment analysis of genes and pathways. The GeneMania and string databases were then used for protein interaction analysis. Results: The GSE1843 dataset was obtained in the GEO database, and three pathways significantly associated with cirrhosis and 13 differential genes enriched in three pathways were screened. Text mining revealed that 11 differential genes were directly associated with cirrhosis. The other two were indirectly linked by other genes. The screened genes and known gene formation networks were discovered by the GeneMania tool. Conclusion: $\mathrm{CDH} 2$ and COL1A1 may be important target genes for cirrhosis.
\end{abstract}

\section{Keywords}

Cirrhosis, Gene Chip, Sinusoidal Endothelial Cells, Bioinformatics

\section{Introduction}

Cirrhosis is the intermediate pathological process of chronic liver disease, which will turn into liver failure and liver cancer if not controlled and reversed [1]. Hepatitis B virus (HBV) is the main cause of liver cirrhosis in China [2]. About 2 billion people in the world have been infected with the HEPATITIS $B$ virus (HBV), 240 million of whom are chronic HBV infected. About 650,000 people die every year from diseases such as cirrhosis and liver cancer caused by HBV 
infection [3]. Liver sinusoidal Phagocytic cells (LSEC) are the main cells constituting hepatic sinusoidal blood sinusoidal sinus; fenestra and basal membrane are characteristic morphological characteristics; fenestra ACTS as a barrier during material exchange [4]. Under the action of chronic liver injury factors, a continuous basal membrane will form at the junction between LSEC and hepatocytes, which will damage the liver microcirculation and accompany the disappearance of window holes. LSEC is closely related to liver diseases [5]. Currently, there are no specific drugs for the treatment of cirrhosis, mainly because the pathogenesis of the disease is very complex, there is no appropriate target for targeted therapy. In this study, gene chips of hepatic sinuses endothelial cells of cirrhotic rats were obtained from the GEO database to screen differential genes, and enrichment pathways, functions, and interactions of differential genes were analyzed through the DAVID GeneMania database, to provide a scientific basis for in-depth research on the molecular mechanism of cirrhosis.

\section{Material and Methods}

\subsection{Acquisition and Analysis of Data Set}

Taking Sinusphagoid Cells as the keyword and expression profiling by Array as the research type restriction, we search-relevant gene chip information in the GEO database and obtain GSE1843 [6] data set, which is derived from the Center for Biochemical and Molecular Genetics in Barcelona, Spain, and platform GPL341.

\subsection{Screening of Differential Genes}

GEO2R [7], an online analysis tool from the GEO database, is a data analysis tool based on the R language that was created in 2012 to perform statistical analysis to screen out differential genes. In this study, liver sinusoidal endothelial cells of cirrhotic rats and normal rat sinusoidal endothelial cells were used as experimental group and control group respectively to analyze the differentially expressed genes in the samples of the two groups. The differentially expressed genes were screened by 0.05 and the differentially expressed multiple of 4 .

\subsection{Bioinformatics Analysis of Differential Genes}

Gene and pathway function annotation was carried out by DAVID [8] (https://david.ncifcrf.gov/), text mining was carried out by COREMINE, and protein interaction analysis was carried out by GeneMania [9] (https://www.genemania.org/).

\section{Consequence}

\subsection{Gene Analysis of Hepatic Sinusoidal Endothelial Cells in Cirrhotic Rats}

Firstly, background correction and standardization were carried out for the chip data of each sample. As can be seen from Figure 1, the chip data after processing 


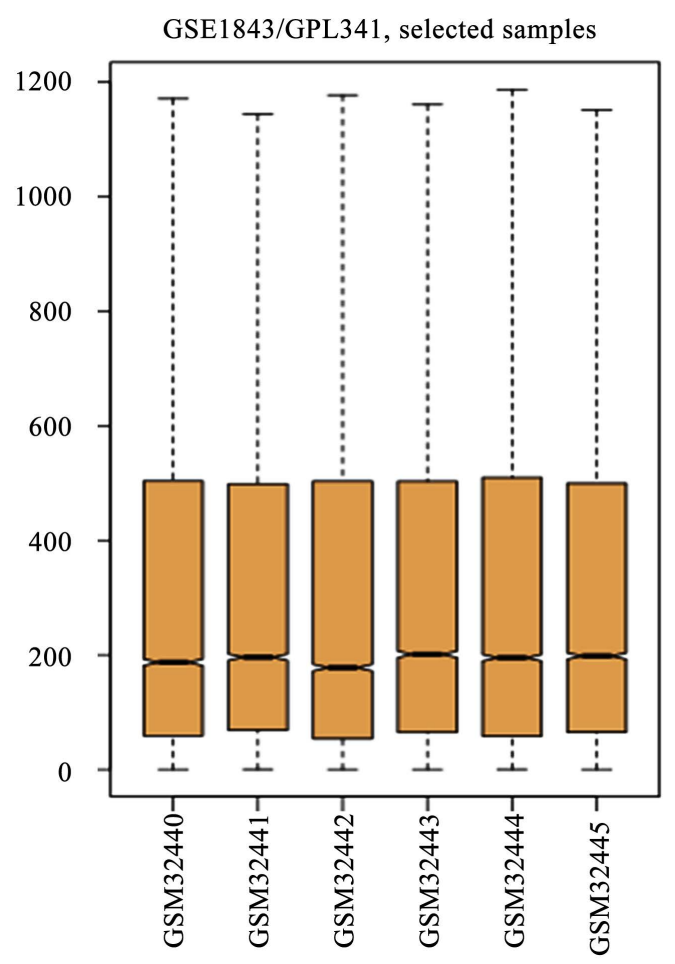

Figure 1. Box chart from processing chip data of sample.

had good distribution. There are 15,924 genes in the GSE1843 dataset, and we call them $\mathrm{P}<0.01$ and diff. 4 were used as screening conditions. Without encoding genes and duplicating genes, we obtained 120 differentially expressed genes, including 100 up-regulated genes and 20 down-regulated genes.

\subsection{Pathway Analysis of Differentially Expressed Genes}

120 differentially expressed genes were input into DAVID database, and the signaling pathways enriched with differentially expressed genes could be obtained by KEGG analysis, according to $\mathrm{P}<$ Three pathways with significantly enriched genes were screened out, as shown in Table 1.

\subsection{Interaction Analysis of Differentially Expressed Genes}

Enrichment on the three pathways of 17 genes has four is repeated, so we focus on the 13 genes, Protein digestion and absorption on the enrichment of the gene for Col1a1 Col1a2 Col5a1 Col6a3 Slc8a1; The genes enriched on ECM-receptor are Col1a1, Col1a2, Col5a1, Col6a3, and Lamb1; The genes abundant on Cell molecules (CAMs) are Alcam, Cdh2, Cldn3, Cldn5, Ncam1, Nrxn1, and Selp.

CARMINE was used for text mining to further explain the correlation between 13 different genes and cirrhosis diseases. The keywords of each gene of cirrhosis were searched, and it was found that in addition to ALCAM NRXN1, the other 11 genes were directly related to the disease of cirrhosis, and these genes also had interaction, for example, COL1A1 and COL1A2 had significant interaction, as shown in Figure 2. 


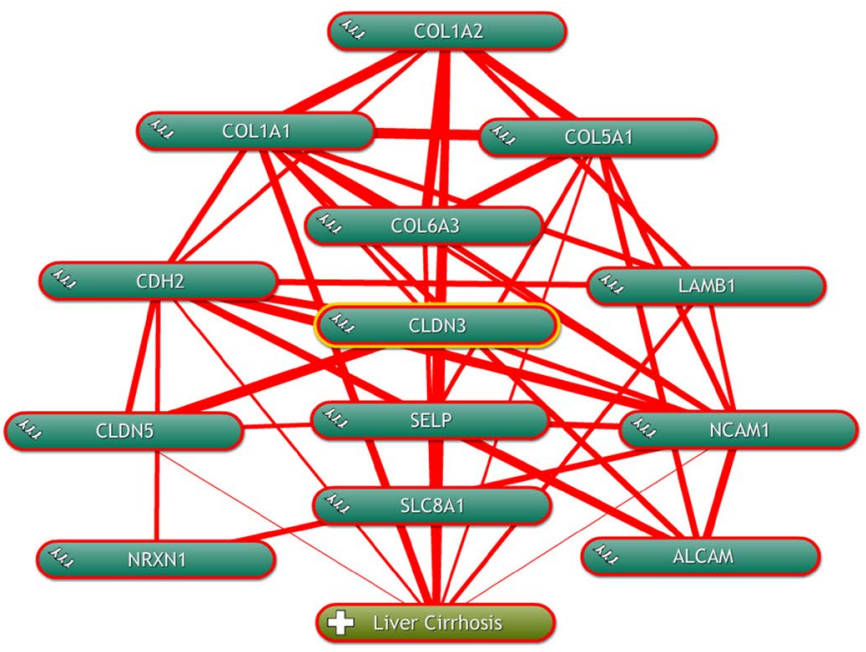

Figure 2. Literature co-occurrence between differential genes and cirrhosis.

Table 1. Pathway analysis of differentially expressed genes.

\begin{tabular}{rcc}
\hline Pathway name & Number of genes & $\mathrm{P}$ \\
\hline Protein digestion and absorption & 5 & $2.9 \times 10^{-3}$ \\
ECM-receptor & 5 & $2.9 \times 10^{-3}$ \\
Cell adhesion molecules (CAMs) & 7 & $9.4 \times 10^{-4}$ \\
\hline
\end{tabular}

\subsection{Differential Genes Interact with Known Genes}

Literature search was conducted on PubMed with cirrhosis and gene as keywords, and every relevant literature was screened and reviewed. Eight genes were found to be closely related to cirrhosis, and GeneMania was used to analyze the interaction between differential genes and known genes, as shown in Figure 3. It can be seen from the figure that the differentiated genes are directly or indirectly related to the known genes, forming a relational network, indicating that these genes are functionally correlated, indicating that the differentiated genes may also be closely related to cirrhosis. $\mathrm{CDH} 2$ interacts directly with EGFR, HGF, and IGF1, SLC8A1 and NCAM1, and ALCAM interacts directly with EGFR and GFAP, COL5A1 interacts directly with EGFR and IGF1, COL6A1 interacts directly with HGF and IGF1, and ALCAM interacts directly with EGFR and BCL2. Different genes also interact with each other. For example, COL1A1 and COL1A2 SELP LAMB1 CLDN5 SLC8A1 COL5A1 COL6A3 have a direct effect. The bar chart is made according to the number of genes directly interacting between different genes, as shown in Figure 4.

\section{Discussion}

Bioinformatics is a new subject that is formed by the cross of many subjects. Bioinformatics data mining at the molecular level provides a new direction for the study of molecular mechanisms of various diseases. In this study, gene chips related to liver cirrhosis in the GEO database were searched, mRNA expression 

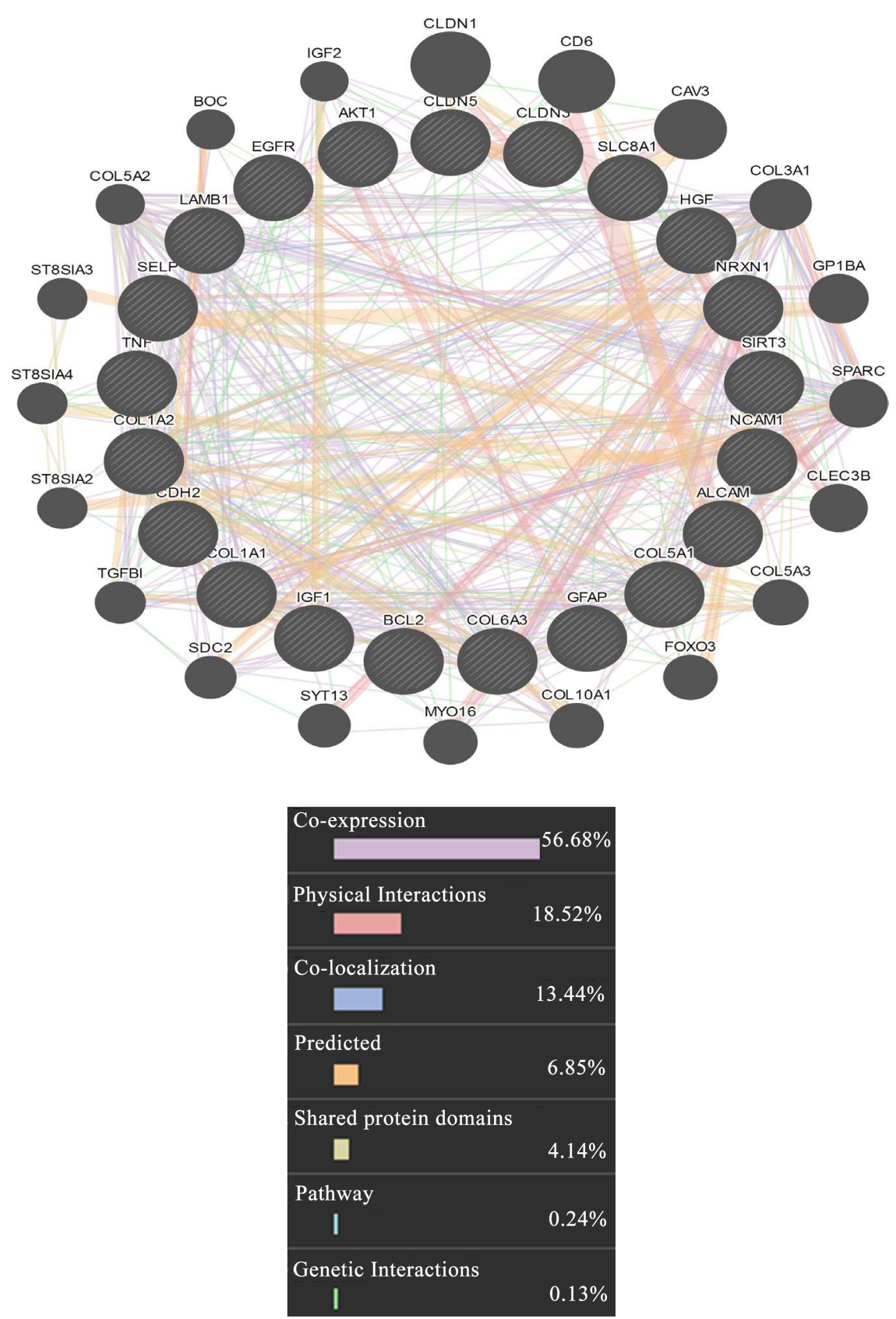

Figure 3. Interaction diagram between known genes and screened genes.

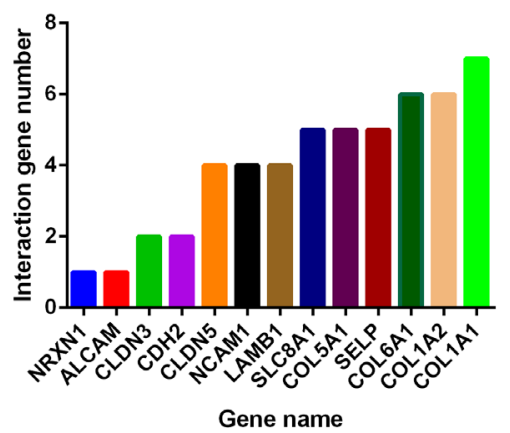

Figure 4. Gene interaction in bar. 
profiles were analyzed, differential genes were screened out, and genes and pathways related to liver cirrhosis were further mined through biosignal pathway enrichment text mining protein interaction analysis methods. Cirrhosis is a chronic progressive disease, and Friedman [10] believes that cirrhosis, like fibrosis, can be reversed. The pathogenesis of cirrhosis is very complex. At present, no marker can accurately reflect the occurrence and development of the disease except pathological examination, so it is very necessary to study the pathogenesis at the molecular level. This study is based on a GEO database mining differences in cirrhosis of the liver sinus endothelial cell genetic data sets, use all kinds of bioinformatics database and software analysis, selected three pathways associated with liver cirrhosis was significantly protein digestion and absorption of ECM receptor pathways in cellular adhesive molecular pathways and enrichment of 13 of the differences in these three pathways genes, including $\mathrm{CDH} 2$ and COL1A1 gene may be important targets of cirrhosis of the liver disease.

Three significant correlation on the path of 13 different genes, the discovery of literature retrieval, is rare in most of the genes in cirrhosis of the liver, such as Wang Junhua [11], the study found in the liver of mice injected foam ball larva of liver fibrosis model is set up, in the extracellular matrix (ECM) accumulation in the process, the detection COL1A1 expression increased significantly, COL1A1 and closely related to liver fibrosis, and liver fibrosis and cirrhosis of the liver is a chronic liver disease in a different period, the performance of COL1A1 and cirrhosis are likely to exist close ties. Retinoic acid can inhibit the activation of hepatic stellate cells and regulate the expression of TGF-1. Studies by $\mathrm{Fu} \mathrm{Yu}$ et al. found that the expression of TGF-1 was down-regulated and the expression of COL1A1 was decreased when retinoic acid was used in rats with chronic alcoholic liver injury, indicating that retinoic acid can protect the liver to a certain extent [12] [13]. Epithelium mesenchymal transition (EMT) has become a hot topic in the study of tumor invasion and metastasis [14]. Cao Guangxin et al. found that EMT expression in bladder cancer tissues was significantly higher than that in normal bladder tissues, and upregulation of $\mathrm{CDH} 2$ was one of the markers of EMT [15] [16]. Studies on CDH2 and cirrhosis have not yet been reported. Whether $\mathrm{CDH} 2$ can be used as a marker molecule for the development of cirrhosis remains to be further studied.

To sum up, this study by GEO database mining a gene chip bioinformatics analysis, selected three liver cirrhosis was significantly related signaling pathways, three signaling pathways enriched in 13 different genes, including $\mathrm{CDH} 2$ and COL1A1 may be important target genes, and molecular mechanism of liver cirrhosis, using bioinformatics analysis method can be carried out on the data of gene chip analysis and screening again, thus efficiently on a large scale for biological internal information, it provides some new mentality on the molecular mechanism for the study of liver cirrhosis. However, only one set of related gene chips was found, which limited the screening of different genes. Therefore, it is necessary to obtain the latest chip for analysis and conduct experimental verification of the screened different genes. 


\section{Conflicts of Interest}

The authors declare no conflicts of interest regarding the publication of this paper.

\section{References}

[1] Lu, F.M. and Zhuang, H. (2009) Management of Hepatitis B in China. Chinese Medical Journal, 122, 3-4.

[2] Wang, F.S., Fan, J.G., Zhang, Z., et al. (2014) The Global Burden of Liver Disease: The Major Impact of China. Hepatology, 60, 2099-2108. https://doi.org/10.1002/hep.27406

[3] Lozano, R., Naghavi, M., Foreman, K., et al. (2012) Global and Regional Mortality from 235 Causes of Death for 20 Age Groups in 1990 and 2010: A Systematic Analysis for the Global Burden of Disease Study 2010. The Lancet, 380, 2095-2128. https://doi.org/10.1016/S0140-6736(12)61728-0

[4] Knolle, P.A., Bottcher, J. and Huang, L.R. (2015) The Role of Hepatic Immune Regulation Systemic Immunity to Viral Infection. Medical Microbiology and Immunology, 204, 21-27. https://doi.org/10.1007/s00430-014-0371-0

[5] Li, S.M., Li, G.Y., Peng, Y. and Zhao, T.J. (2016) Effects of Hepatic Sinusoidal Endothelial Cells on Hepatic Fibrosis and Its Regulatory Mechanism. Journal of Chongqing Medical University, 41, 624-627.

[6] Hugues, S., Morales-Ruiz, M., Fernandez-Varo, G., Ros, J., et al. (2005) Microarray Analysis of Endothelial Differentially Expressed Genes in the Liver of Cirrhotic Rats. Gastroenterology, 129, 1686-1695. https://doi.org/10.1053/j.gastro.2005.09.006

[7] Barrett, T., Wilhite, S.E., Ledoux, P., et al. (2013) NCBI GEO: Archive for Functional Genomics Data Sets-Update. Nucleic Acids Research, 41, D991-D995. https://doi.org/10.1093/nar/gks1193

[8] Huang da, W., Sherman, B.T. and Lempicki, R.A. (2009) Bioinformatics Enrichment Tools: Paths toward the Comprehensive Functional Analysis of Large Gene Lists. Nucleic Acids Research, 37, 1-13. https://doi.org/10.1093/nar/gkn923

[9] Dennis, G., Sherman, B.T., Hosack, D.A., et al. (2003) DAVID: Database for Annotation, Visualization, and Integrated Discovery. Genome Biology, 4, P3. https://doi.org/10.1186/gb-2003-4-5-p3

[10] Friedman, S.L. (2005) Liver Fibrosis: New Frontiers in Liver Disease (English). Compilation of Papers of the First International Conference on Liver Disease of Integrated Traditional Chinese and Western Medicine, Shanghai, 8-10.

[11] Wang, J.H., Wei, X.F., Zhang, C.S., et al. (2012) Expression and Significance of Collagen Gene in the Liver of Mice in the Middle Stage of Metacercariae Infection. Journal of Pathogen Biology, 7, 653-656.

[12] Fu, Y., Dan, Z.-L., Tang, W.-X. and Yan, W. (2006) Effect of All-Trans Retinoic Acid on Expression of TGF- $\beta 1$, CTGF and Colla1 in Chronic Alcoholic Liver Disease in Rats. World Chinese Journal of Digestology, 14, 179-183.

[13] Davis, B.H., Kramer, R.T. and Davidson, N.O. (1990) Retinoic Acid Modulates Rat Ito Cell Proliferation, Collagen, and Transforming Growth Factor-Beta Production. Journal of Clinical Investigation, 86, 2062-2070. https://doi.org/10.1172/JCI114943

[14] Baumgart, E., Cohen, M.S., Silva Neto, B., et al. (2007) Identification and Prognostic Significance of an Epithelial-Mesenchymal Transition Expression Profile in Human Bladder Tumors. Clinical Cancer Research, 13, 1685-1694. 
https://doi.org/10.1158/1078-0432.CCR-06-2330

[15] Cao, G.X., Zhang, Y.X. and Wang, X.L. (2014) Expression of Genes Related to Epithelial-Stromal Differentiation in Bladder Cancer. Jiangsu Medical Journal, 40, 2061-2062.

[16] Huber, M.A., Kraut, N. and Beug, H. (2005) Molecular Requirements for Epithelial-Mesenchymal Transition during Tumor Progression. Current Opinion in Cell Biology, 17, 548-558. https://doi.org/10.1016/j.ceb.2005.08.001 